\title{
The expression of Drosha, DGCR8, Dicer and Ago-2 genes are upregulated in human umbilical vein endothelial cells under hyperglycemic condition
}

\author{
Farhad Ghadiri Soufi ${ }^{1}$, Ali Akbar Poursadegh Zonouzi ${ }^{2}$, Ebrahim Eftekhar ${ }^{1}$, Kamila Kamali ${ }^{3}$, \\ Sara Aghakhani Chegeni ${ }^{4}$, Ahmad Poursadegh Zonouzi ${ }^{5}$
}

\begin{abstract}
${ }^{1}$ Molecular Medicine Research Center, Hormozgan Health institute, Hormozgan University of Medical Sciences, Bandar Abbas, Iran; ${ }^{2}$ Department of Genetics and Medical Biotechnology, Faculty of Medicine, Hormozgan University of Medical Sciences, Bandar Abbas, Iran; ${ }^{3}$ Larestan University of Medical Sciences, Larestan, Iran; ${ }^{4}$ Student Research Committee, Hormozgan University of Medical Sciences, Bandar Abbas, Iran; 5Biotechnology Research Center, Tabriz University of Medical Sciences, Tabriz, Iran

E-mail:kamali_k67@yahoo.com
\end{abstract}

Objectives. It has been shown that dysregulation of miRNAs expression contributes to the pathogenesis and progression of the diabetes and diabetes-related complications. Drosha, DGCR8, Dicer, and Ago-2 are involved in the miRNA maturation. The aim of the present study was to investigate the mRNA expression levels of these genes in the human umbilical vein endothelial cells (HUVECs) under hyperglycemic condition.

Methods. HUVECs were cultured in normo- $(5 \mathrm{mM})$ and hyperglycemic $(25 \mathrm{mM})$ conditions for $24 \mathrm{~h}$. As osmotic control, cells were treated with D-mannitol $(25 \mathrm{mM}$, for $24 \mathrm{~h})$. The mRNA expression levels of Drosha, DGCR8, Dicer and Ago-2 were evaluated using quantitative real-time PCR.

Results. The expression level of Drosha, DGCR8, Dicer, and Ago-2 were increased in hyperglycemic HUVECs compared to the control group.

Conclusion. Our results show that under hyperglycemic condition, expression of genes involved in the miRNA maturation was significantly increased in HUVECs. Upregulation of these genes may have role in diabetic complications through the dysregulation of the miRNA expression.

Key words: hyperglycemia, Drosha, DGCR8, Dicer, Ago-2, human umbilical vein endothelial cells

MiRNAs are small 2 21-nucleotide noncoding RNAs (ncRNAs) that delicately orchestrate the expression of protein-coding genes at the post-transcriptional level through targeting mRNAs in a sequence-specific manner by triggering translational repression or, more commonly, degradation of the target mRNAs (Di Leva et al. 2006; Guay and Regazzi 2013; Park et al. 2013). The mRNAs targeted by miRNAs are involved in a wide variety of important biological processes such as DNA repair, cell cycle progression, chromatin structure remodeling, im- mune responses, and inflammation (Aure et al. 2013; McClelland and Kantharidis 2014). Not surprisingly, aberrant expression of these small regulatory switches may have a big effect on the normal physiological processes (Di Leva et al. 2006). Over the past several years, it has become clear that altered miRNA expression contributes to the development and progression of various human disorders, especially the diabetes (Park et al. 2013; McClelland and Kantharidis 2014). Although the basic mechanisms of altered miRNA expression profile are now reasonably well 
understood, in malignances understanding the ways in which miRNA expression and activity are disrupted in diabetes are still under the progress (McClelland and Kantharidis 2014; Lin and Gregory 2015). Dysregulation of miRNA biogenesis machinery component(s) have been suggested, at least in part, as a possible molecular cause of miRNAome alteration (Lin and Gregory 2015). The biogenesis of miRNAs is a stepwise process that requirements multiple ribonuclease (RNase) III family enzymes and cofactors (Guay and Regazzi 2013). The miRNA genes initially are transcripted by RNA polymerase II to generate a primary miRNA (pri-miRNA) (Lin and Gregory 2015). To form pre-miRNA, the pri-miRNA cleaves by Drosha, as a catalytic core of microprocessor complex, through composition with Digeorge Syndrome Critical Region 8 (DGCR8) which act as a "molecular ruler" by recognizing the cleavage site within the primiRNA (Fardmanesh et al. 2016). Subsequently, premiRNA transferred by exportin 5/RanGTP complex into cytoplasm through the nuclear pore complex (Lin and Gregory 2015). In cytoplasm, Dicer cleaves pre-miRNA and release a $\sim 22$ nucleotide duplex containing two strands, termed miRNA/miRNA ${ }^{*}$ duplex (Lin and Gregory 2015; Poursadegh Zonouzi et al. 2015). Finally, one strand of the miRNA/miRNA* duplex is loaded onto an Argonaute (Ago) protein, which acts as a catalytic engine of a large multi-proteinic assembly called RNA-induced silencing complex (RISC) (Lin and Gregory 2015). The miRNARISC complex binds to complementary sequence within target mRNA and regulates gene expression (Lin and Gregory 2015).

The expression pattern of the miRNA biogenesis machinery components in diabetes has been poorly understood and it raises the question whether dysregulation of these components can be or not involved in diabetes-related complications. In an attempt to address this question, we designed in this study to investigate the mRNA expression pattern of Drosha, DGCR8, Dicer, and Ago-2 genes in the human umbilical vein endothelial cells (HUVECs) under hyperglycemic condition.

\section{Materials and Methods}

Cell Culture. The human umbilical vein endothelial cells (HUVECs) were obtained from the National Cell Bank of Iran (NCBI, Pasteur Institute, Tehran) and cultured in endothelial cell growth medium, EGM-2 Bullet kit (Lonza, Basel, Switzerland), according to the manufacturer's recommendations. Cells were incubated in humidified $5 \% \mathrm{CO}_{2}$ atmosphere at $37^{\circ} \mathrm{C}$ and were passaged on reaching confluence using $0.25 \%$ trypsin-EDTA (Gibco, Grand Island, NY, USA) every 3-4 days. All reagents were purchased from Sigma-Aldrich (St. Louis, MO) unless otherwise specified.

Exposure of HUVECs to hyperglycemia. HUVECs at a density of $3 \times 10^{5}$ cells were seeded in $6-\mathrm{cm}$ tissue culture dishes under standard culture condition for $24 \mathrm{~h}$. Cells were treated with $20 \mathrm{mM} \mathrm{D}$-glucose as hyperglycemic group and $5 \mathrm{mM} \mathrm{D}$-glucose as control, for $24 \mathrm{~h}$. As osmotic control, cells were treated with $20 \mathrm{mM}$ D-manitol. The experiments were done in triplicates and repeated three times.

RNA isolation and cDNA synthesis. Total RNA was isolated from HUVECs using miRNeasy Mini Kit (Qiagen) according to the manufacturer's instructions. The quality and quantity of extracted RNA were confirmed by agarose electrophoresis and NanoDrop ${ }^{\circledR}$ ND-1000 Spectrophotometer (Thermo Scientific, USA), respectively. Subsequently, total RNA $(1 \mu \mathrm{g})$ was reversely transcribed to cDNA using miScript II RT cDNA synthesis Kit (Qiagen), following the manufacturer's protocol.

Quantitative Real-time PCR analysis. Real-time PCR was conducted in triplicate using gene-specific primers and miScript SYBR Green PCR Kit (Qiagen) by the Rotor-GeneTM 6000 system (Corbett Research, Australia) according to the manufacturer's instructions. Primer sequences are shown in Table 1. Each reaction mixture contained equal amount of cDNA, master mix 2X, RNase free water, and 10 pmol of each primer pairs for Drosha, DGCR8, Dicer, Ago-2 and $\beta$-actin in a final volume of $25 \mu$ l. Initial denaturation at $94^{\circ} \mathrm{C}$ for $30 \mathrm{~s}$ was followed by 45 cycles (for Drosha, DGCR8, Dicer and Ago-2) and 35 cycles (for $\beta$-actin) of denaturation at $94^{\circ} \mathrm{C}$ for $5 \mathrm{~s}$, annealing at specific temperatures (Table 1) for each primer pairs for $15 \mathrm{~s}$, extension at $72^{\circ} \mathrm{C}$ for $10 \mathrm{~s}$. Relative amount of mRNA was calculated using $2^{-\Delta \Delta C t}$ method and normalized to level of beta actin.

Statistical analysis. The data are expressed as mean \pm SD. Differences between mRNA expression levels of two studied groups were determined by Mann-Whitney $U$ test. The level of significance was set at $\mathrm{p}<0.05$. Statistical analyses were done using the SPSS software (SPSS for Windows, version 12.0).

\section{Results}

We investigated the mRNA expression level of Drosha, DGCR8, Dicer, and AGO-2 in HUVECs using quantitative real-time PCR method under hyperglycemic condition. Our results demonstrated that 
Table 1

Sequence of primers used in quantitative real-time PCR and the annealing and melting temperature of each primer

\begin{tabular}{llcc}
\hline Gene & Sequence of primers & Annealing temperature $\left({ }^{\circ} \mathrm{C}\right)$ & Melting temperature $\left({ }^{\circ} \mathbf{C}\right)$ \\
\hline \multirow{2}{*}{ 3-actin } & F 5'-CAGCCATGTACGTTGCTATCCAGG-3' & 60 & 63 \\
& R 5'-AGGTCCAGACGCAGGATGGCATG-3' & & 66 \\
\multirow{2}{*}{ Drosha } & F 5'-AGCCCTGGTGCCTGAGGAGGAGAT -3' & 68.2 & 69.5 \\
& R5'-TGCAGGGCGTATCCCAAAGTGGAC-3' & & 67 \\
\multirow{2}{*}{ DGCR8 } & F 5'-CAAGCAGGAGACATCGGACAAG-3' & 61.9 & 62 \\
& R 5'-CACAATGGACATCTTGGGCTTC-3' & & 60 \\
\multirow{2}{*}{ Dicer } & F 5'-AGCCCCAGCCCAGCGATGAA-3' & 64.2 & 66 \\
& R 5'-GTCCAGGATTGGGGCCAAGAGTCC-3' & & 66 \\
Ago2 & F 5'-TGCAGGCGTTACACGATGC-3' & 60.3 & 61 \\
\hline & R 5'-TACCTCATGGATGGCAAGTGC-3' & & 60 \\
\hline
\end{tabular}

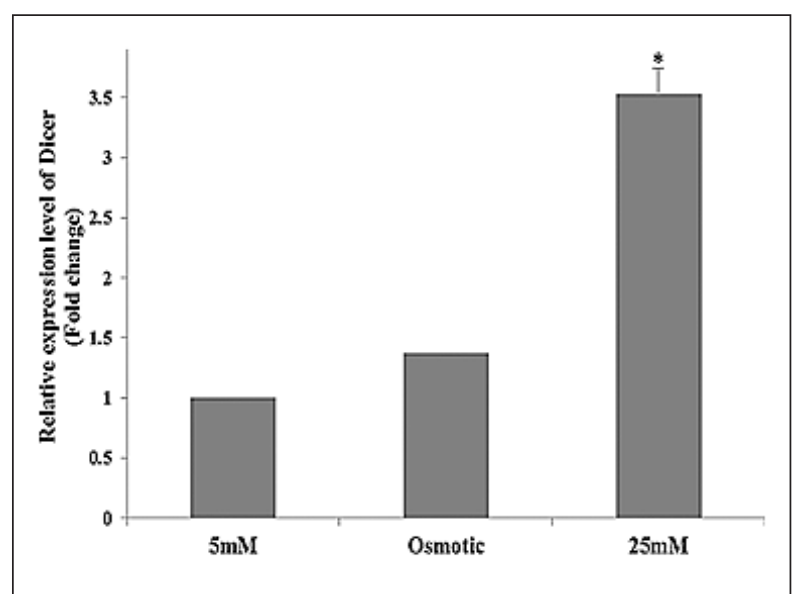

Figure 1. The mRNA expression levels of Dicer in HUVECs exposed to $5 \mathrm{mM}$ D-glucose (normal) and $25 \mathrm{mM}$ D-glucose (hyperglycemic) and $5 \mathrm{mM} \mathrm{D}$-glucose $+20 \mathrm{mM} \mathrm{D}$-mannitol (osmotic control). Bars represent the Mean $\pm \mathrm{SD}$. ${ }^{*} \mathrm{p}<0.05$ vs. $5 \mathrm{mM}$ D-glucose.

the mRNA expression level of Drosha, DGCR8, Dicer, and AGO-2 genes were significantly upregulated after incubation with $25 \mathrm{mM}$ glucose in HUVECs. On the other hand, we did not detect any significant changes in expression level of these genes in osmotic controls (Figures 1-4). The mRNA expression levels of Dicer and Drosha genes were significantly upregulated (more than threefold) in the hyperglycemic condition compared with $5 \mathrm{mM}$ (Figures 1, 2). Moreover, the mRNA expression levels of Ago-2 and DGCR8 genes were significantly overexpressed (more than fourfold) in the hyperglycemic condition (Figures 3,4 ). Under hyperglycemic condition, the highest expression levels were observed in DGCR8, Ago-2, Drosha, and Dicer.

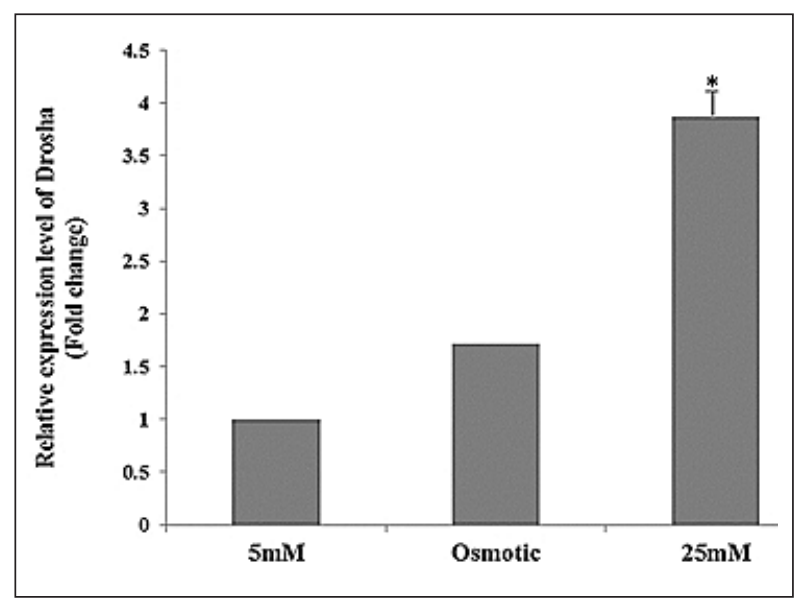

Figure 2. The mRNA expression levels of Drosha in HUVECs exposed to $5 \mathrm{mM}$ D-glucose (normal) and $25 \mathrm{mM}$ D-glucose (hyperglycemic) and $5 \mathrm{mM} \mathrm{D}$-glucose $+20 \mathrm{mM} \mathrm{D}$-mannitol (osmotic control). Bars represent the Mean $\pm \mathrm{SD}$. ${ }^{*} \mathrm{p}<0.05$ vs. $5 \mathrm{mM}$ D-glucose.

\section{Discussion}

The fundamental role of various miRNAs in the regulation of insulin synthesis and secretion, insulin resistance of skeletal muscle, hepatic and adipose tissues, and $\beta$-cell fate, and pancreatic mass formation have previously been documented (Melkman-Zehavi et al. 2011; Kaspi et al. 2014; Bhattacharya et al. 2015). Therefore, dysregulation of miRNAs expression contributes to genesis and progression of diabetes and diabetes-related complications (Guay et al. 2011; Kantharidis et al. 2011). On the other hand, as indicated earlier, Drosha, DGCR8, Dicer and Ago-2 are involved in miRNA maturation. Hence, we investigated the mRNA expression levels of these genes in HU- 


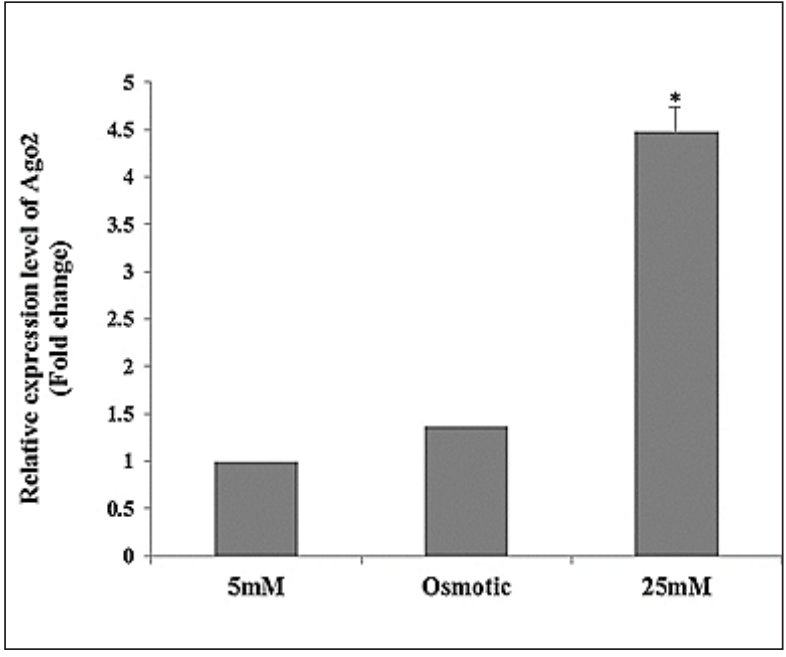

Figure 3. The mRNA expression levels of Ago2 in HUVECs exposed to $5 \mathrm{mM}$ D-glucose (normal) and $25 \mathrm{mM}$ D-glucose (hyperglycemic) and $5 \mathrm{mM} \mathrm{D}$-glucose $+20 \mathrm{mM} \mathrm{D}$-mannitol (osmotic control). Bars represent the Mean $\pm \mathrm{SD}$. ${ }^{*} \mathrm{p}<0.05$ vs. 5 mM D-glucose.

VECs under hyperglycemic condition. Our findings revealed that the mRNA expression levels of Drosha, DGCR8, Dicer and Ago-2 genes were significantly increased in hyperglycemic condition compared with normoglycemic $(5 \mathrm{mM})$. This is in the harmony with our previous data reporting that the mir-146a expression level are upregulated in HUVECs under hyperglycemic condition (Kamali et al. 2016).

The role of the miRNA machinery components in progression of diabetes is corroborated by the fact that deletion and/or alteration in the expression levels of the components lead to defective insulin secretion and impaired development of pancreatic $\beta$-Cell (Kalis et al. 2011; Tattikota et al. 2013; Chavali et al. 2014; Rahimi et al., 2015). In accordance with our findings, Rahimi et al. (2015) were found dysregulation of miRNA machinery components in patients with gestational diabetes mellitus. They have reported that the expression levels of Drosha, Dicer and DGCR8 were upregulated in patients with gestational diabetes mellitus compared to the control group (Rahimi et al. 2015). Chavali et al. (2014) have demonstrated that the expression of Dicer is upregulated in insulin mutant (Ins2+/-) Akita mouse models. It has also been documented that beta-cell specific deletion of Dicer in mouse model leads to a progressive impairment in insulin secretion, glucose homeostasis, and diabe-

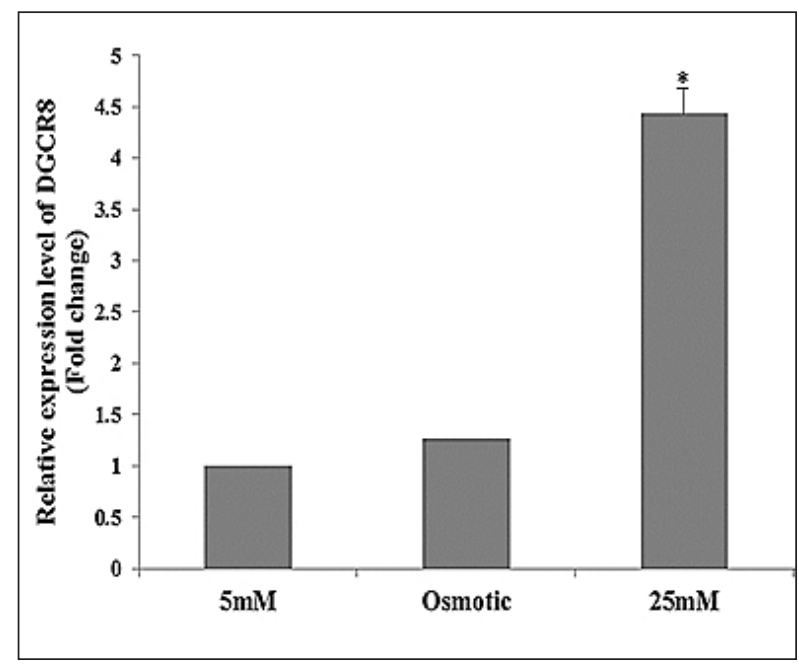

Figure 4. The mRNA expression levels of DGCR8 in HUVECs exposed to $5 \mathrm{mM}$ D-glucose (normal) and $25 \mathrm{mM}$ D-glucose (hyperglycemic) and $5 \mathrm{mM} \mathrm{D}$-glucose $+20 \mathrm{mM} \mathrm{D}$-mannitol (osmotic control). Bars represent the Mean $\pm \mathrm{SD}$. ${ }^{\star} \mathrm{p}<0.05$ vs. $5 \mathrm{mM}$ D-glucose.

tes development (Kalis et al. 2011). The role of Ago-2 in the systemic release of proteins from MIN6 betacell line model has been reported by Tattikota et al. (2013). In the light of these studies and our data, dysregulation of the miRNA machinery component(s) may be involved in the pathogenesis of the diabetes mellitus. Of note, in parallel with a small dysfunction of the miRNA machinery component(s), maturation of large body of miRNAs is disrupted, which in turn affects the broader range of fundamental cellular processes involved in the maintenance of the physiologic steady state.

In conclusion, the results of the present study indicate that Drosha, DGCR8, Dicer, and Ago-2 genes were significantly upregulated in HUVECs under hyperglycemic condition. Increased expression of these miRNA maturation components may have a role in diabetic complications through the dysregulation of the miRNAs expression.

\section{Acknowledgments}

This study was supported by the grant of Molecular Medicine Research Center, Hormozgan University of Medical Sciences, Bandar Abbas, Iran. The authors wish to thank all the participants who were taking part in this study. 


\section{References}

Aure MR, Leivonen SK, Fleischer T, Zhu Q, Overgaard J, Alsner J, Tramm T, Louhimo R, Alnæs GI, Perala M, Busato F, Touleimat N, Tost J, Børresen-Dale AL, Hautaniemi S, Troyanskaya OG, Lingjærde OC, Sahlberg KK, Kristensen VN. Individual and combined effects of DNA methylation and copy number alterations on miRNA expression in breast tumors. Genome Biol 14, R126, 2013.

Bhattacharya S, Aggarwal R, Singh VP, Ramachandran S, Datta M. Downregulation of miRNAs during delayed wound healing in diabetes: Role of Dicer. Mol Med 2015.

Chavali V, Tyagi SC, Mishra PK. Differential expression of dicer, miRNAs, and inflammatory markers in diabetic Ins2+/- Akita hearts. Cell Biochem Biophys 68, 25-35, 2014.

Di Leva G, Calin GA, Croce CM. MicroRNAs: fundamental facts and involvement in human diseases. Birth Defects Res C Embryo Today 78, 180-189, 2006.

Fardmanesh H, Shekari M, Movafagh A, Alizadeh Shargh S, Poursadegh Zonouzi AA, Shakerizadeh S, Poursadegh Zonouzi A, Hosseinzadeh A. Upregulation of the double-stranded RNA binding protein DGCR8 in invasive ductal breast carcinoma. Gene 581, 146-151, 2016.

Guay C, Roggli E, Nesca V, Jacovetti C, Regazzi R. Diabetes mellitus, a microRNA-related disease? Transl Res 157, 253-264. 2011.

Guay C, Regazzi R. Circulating microRNAs as novel biomarkers for diabetes mellitus. Nat Rev Endocrinol 9, 513521, 2013.

Kalis M, Bolmeson C, Esguerra JL, Gupta S, Edlund A, Tormo-Badia N, Speidel D, Holmberg D, Mayans S, Khoo NK, Wendt A, Eliasson L, Cilio CM. Beta-cell specific deletion of Dicer1 leads to defective insulin secretion and diabetes mellitus. PLoS One 6, e29166, 2011.

Kamali K, Korjan ES, Eftekhar E, Malekzadeh K, Soufi FG. The role of miR-146a on NF- $\kappa$ B expression level in human umbilical vein endothelial cells under hyperglycemic condition. Bratisl Lek Listy 117, 376-380, 2016.

Kantharidis P, Wang B, Carew RM, Lan HY. Diabetes complications: the microRNA perspective. Diabetes 60, 18321837, 2011.

Kaspi H, Pasvolsky R, Hornstein E. Could microRNAs contribute to the maintenance of $\beta$ cell identity? Trends Endocrinol Metab 25, 285-292, 2014.

Lin S, Gregory RI. MicroRNA biogenesis pathways in cancer. Nat Rev Cancer 15, 321-333, 2015.

McClelland AD, Kantharidis P. microRNA in the development of diabetic complications. Clin Sci (Lond) 126, 95110, 2014.

Melkman-Zehavi T, Oren R, Kredo-Russo S, Shapira T, Mandelbaum AD, Rivkin N, Nir T, Lennox KA, Behlke MA, Dor Y, Hornstein E. miRNAs control insulin content in pancreatic $\beta$-cells via downregulation of transcriptional repressors. EMBO J 30, 835-845, 2011.

Park SY, Jeong HJ, Yang WM, Lee W. Implications of microRNAs in the pathogenesis of diabetes. Arch Pharm Res 36, 154-166, 2013.

Rahimi G, Jafari N, Khodabakhsh M, Shirzad Z, Dogaheh HP. Upregulation of microRNA processing enzymes Drosha and Dicer in gestational diabetes mellitus. Gynecol Endocrinol 31, 156-159, 2015.

Tattikota SG, Sury MD, Rathjen T, Wessels HH, Pandey AK, You X, Becker C, Chen W, Selbach M, Poy MN. Argonaute2 regulates the pancreatic $\beta$-cell secretome. Mol Cell Proteomics 12, 1214-1225, 2013.

Poursadegh Zonouzi AA, Nejatizadeh A, Rahmati-Yamchi M, Fardmanesh H, Shakerizadeh S, Poursadegh Zonouzi A, Nejati-Koshki K, Shekari M. Dysregulated expression of Dicer in invasive ductal breast carcinoma. Med Oncol 32, 203, 2015. 